\title{
Psychopharmacology and people with learning disability
}

\author{
Bill Fraser
}

\begin{abstract}
"These medications (antipsychotic drugs) were originally developed to treat not the mentally handicapped but the mentally ill; those, for example, with schizophrenia, paranoia and other specific conditions, but now the far wider use of these drugs has been challenged because there is evidence that they can produce serious side-effects in addition to the distress already suffered. Future changes in the use of antipsychotic drugs in the UK may come from the Royal College of Psychiatrists or from action in the Law Courts" (Public Eye, BBC2, 1.5.92).
\end{abstract}

The Royal College of Psychiatrists has published guidelines for the use of high doses of neuroleptics following the concern expressed in the media regarding sudden deaths associated with the use of these drugs (Thompson, 1994). This television programme heralded a much more stringent approach to the prescribing of antipsychotic drugs for people with learning disability and an awareness of the power of litigation in the USA to affect practice in the UK.

People with learning disability are one of the most highly medicated populations in our society. Foremost among the medications are psychoactive drugs. Numerous surveys suggest that these medications are used quite liberally for the main purpose of controlling behaviours. Between 20 and $45 \%$ of people with learning disability are on antipsychotic drugs, of which $14-30 \%$ are taking these to control challenging behaviour (Deb \& Fraser, 1994). Discharge from institutions little changes medication levels.

\section{Prescribing for people with learning disability}

Additional problems are presented by people with learning disability. Many of the standard approaches to diagnosis rely on communication capabilities which are often absent in people with learning disability. Also, they often cannot complain of adverse effects. Administration of medication has to take account of the multiple additonal handicaps these people often have-for example, cardiac, renal, and metabolic as well as underlying abnormality of the central nervous system. For people with severe learning disability, medical histories are likely to be very incomplete and informants not always present or capable. Compliance depends on carers' carefulness and attitudes. Additional disorders such as epilepsy can make a patient more vulnerable to adverse drug effects and drug interaction. There is a particular need to record idiosyncratic adverse effects.

The use of psychotropic drugs in individuals with developmental disability must be subject to regular audit (see Box 1). Detailed accounts of the development of audit procedures are described in Einfeld (1990) and Bishop (1992).

Box 1. Audit criteria

Audit requires documented case note evidence that:

Drug treatment forms part of an overall management package

Drug treatment follows baseline mental state examination

Drug treatment follows behavioural analysis

Other factors that may affect the person's behaviour have been considered (see Box 2)

Drug treatments should (except in emergencies) be fully aired in multi-disciplinary meetings involving clients and carers

There is regular monitoring of target symptoms and total treatment package

Bill Fraser (University of Wales College of Medicine, Division of Psychological Medicine, Ely Hospital, Cowbridge Road West, Cardiff CF5 5XE) is a Co-Director of the Welsh Centre for Learning Disabilities and has published widely in the management of developmental disability. He was recently grantholder in a national research and development project into antipsychotic drug reduction packages. Since 1983 he has been Editor of the Journal of Intellectual Disability Research. 
A rational treatment approach should always be based on the existing evidence in the literature. As Healy \& Nutt (1998) point out, there is a lack of evidence on medication prescribing in general with children and people with learning disability. The authors warn against inflexible prescribing ruled by disclaimers in data sheets. The practitioner has to make a calculation of risks and benefits and, if necessary, prescribe 'off-licence'. The guiding principle is that where there is a natural continuity with adulthood conditions (for example, anticonvulsants do not usually have a product licence for use in children), prescribing can proceed in the absence of a product licence. For the elderly, treatment should be started at a lower dose and increased more gradually.

The evidence base for prescribing in learning disability is very deficient. Most importantly, the use of antipsychotic medication in people with learning disbility is not based on randomised controlled trials (RCTs). Even in the case of the psychiatrically ill - antipsychotic medication for schizophrenia there is only one RCT (Duggan \& Brylewski, 1999). Because of limited communication, diagnosis is more reliant on observation, rating scales and carer information. The population with learning disability is a heterogeneous one with great variation. Berney (personal communication, 1999) points out that in every treatment programme, behavioural changes are often attributed to medication changes when these are just a coincidence and may be due to environmental change or maturational and natural psychological change (see Box 2). The efficacy of medication can depend on chance extrinsic factors. Vague treatment objectives are common with people with learning disability, so we do not know whether treatment is aimed at a clinical feature or a mental disorder.

The psychopharmacology of learning disability has been hindered by factors such as the relative

Box 2. Factors that may influence behaviour in a person with learning disability

Undetected physical illness

Undetected psychiatric illness

Drug side-effects, interactions and withdrawal

Underlying brain damage

Communication problems

Undetected neuropsychiatric disorder

Environment over- or under-stimulates the individual

High expressed emotion by family/staff

Life change events, especially loss or abuse Lack of problem-solving ability lack of involvement of psychiatry in learning disability and, up until recently, a reluctance in some quarters to make a psychiatric diagnosis for ideological reasons. The inappropriate blunderbussbehavioural' historical use of psychotropic drugs leading to the $\mathrm{BBC}$ report quoted above has meant that, wrongly, psychotropic medication and behavioural methods are sometimes seen as incompatible. The estimation of benefits and the additional risks for individuals with learning disability involves close cooperation between the care-giver, the individual with learning disability, the community team and the prescribing practitioner. Doing nothing and not prescribing may be a greater risk for the individual. The positive principle of a minimum effective dose is generally accepted (although not for medications such as lithium) and may be combined with drug holidays (e.g. from methylphenidate) - but the risk of these increasing the likelihood of tardive dyskinesia in the case of neuroleptics must be taken into account.

\section{The process of diagnosis}

To assist documentation of reasons for medication, screening instruments for assessing psychopathology - such as the Psychopathology Instrument for Mentally Retarded Adults (Matson, 1988), the Reiss Screen for Maladaptive Behaviour (Reiss, 1988), or the Psychiatric Assessment Schedule for Adults with a Developmental Disability (PAS-ADD) Checklist and Mini-PAS-ADD (Moss et al, 1994) are designed to help staff recognise mental health problems in their patients and make informed referral decisions to diagnose them. The PAS-ADD Checklist is a 'screen only' instrument that requires no training or qualifications; the Mini-PAS-ADD provides professional care staff with a structured framework to collect information on psychiatric symptoms. The PAS-ADD is a semi-structured instrument for the use of respondents who have learning disability and for key informants. It requires a knowledge of psychopathology and training in a semi-structured clinical interview. The full PAS-ADD is increasingly being adopted in Europe as a gold standard for diagnosis of Axis 1 disorders (Moss et al, 1997)

There are problems associated with rating scales. Reliability requires raters to be accurate and there is always a tendency for extreme scores to return towards the mean. Overt behaviours are easier to rate than mood states. The Aberrant Behavior Checklist (Aman et al, 1985) has been tried and tested for over a decade. It was specifically developed to assess drug effects. 
Given a diagnosis of an Axis 1 disorder, the principle is natural continuity - prescribe on the lines one would prescribe for a patient of normal intelligence, taking into account the special considerations about this population mentioned above. It is salutary to be aware that for this population there is often little or no randomised, controlled evidence base to support the use of psychotropic drugs, even for clearly diagnosed disorders. However, it is equally true that there is no RCT to show that these drugs are ineffective in this context. The information so far is largely based on type IV evidence (observational studies) and type $V$ evidence (expert opinion and reports) (classification based on the Bandolier system (http:www.jr2.ox.dc.uk/bandolier/band6/ 66-5.html)). The difficulties increase when one is faced with behavioural problems and no specific diagnosis.

\section{Psychotropic medication for behavioural problems}

Given a clear psychiatric diagnosis, the principles of prescribing are in continuity with prescribing for those of normal intelligence. The problems are with the use of psychotropic drugs for non-specific behavioural disorders. In Clarke et al (1990), those who did not have a diagnosis of psychiatric illness $(36 \%)$ also received psychotropic medication, suggesting that the main indication for the use of psychotropic medication in those people was behavioural problems. However, in some cases the underlying psychiatric illness that caused the behavioural problem remains difficult to diagnose and in other cases behavioural disorders could be perceived as part of a wider behavioural phenotype.

The use of psychotropic drugs for behavioural problems in people with learning disability does not just depend on the individual's behaviour. Factors such as as staff perceptions, environmental factors, staffing ratios, and administrative and treatment philosophies also influence the use of medication (Ahmed et al, 1999). Aggressive behaviour is the strongest predictor of the use of psychotropic drugs. Thus, staff perceptions of behavioural problems, self-injurious behaviour and physical aggression are more influential factors for maintenance of psychotropic drug treatment than the reasons for which the drugs were prescribed initially.

A recent Cochrane systematic review (Brylewski \& Duggan, 1998), commissioned to determine the effectiveness of antipsychotic medication for people with learning disability and challenging behaviour, found that only three RCTs could be included in the analyses. These provided no evidence of whether antipsychotic medication controls challenging behaviour in adults with learning disability. The authors state that in the absence of trial evidence, clinicians will have to continue to base prescribing on clinical experience and judgement.

\section{Neuroleptics}

Antipsychotic drugs are divided into 'typical' (the older drugs such as chlorpromazine) and 'atypical' (newer drugs such as risperidone and olanzapine). The atypicals are associated with little or no extra pyramidal side-effects (EPS). The two principal dopamine receptors are dopamine types 1 and $2\left(D_{1}\right.$ and $D_{2}$ ). Receptors $D_{3}$ and $D_{4}$ are also implicated in antipsychotic effect. Neuroleptics largely block dopamine transmission, and atypicals also affect the 5-HT system.

The $D_{1}$ receptors have a particular role to play in aggression and self-injurious behaviour. Newer (atypical) 'cleaner' neuroleptics - for example, clozapine, which has a low $\mathrm{D}_{2}$ receptor occupancy, particularly in the striatum but not in the limbic structure (which probably explains the low frequency of EPS) - are also effective, in open trials, with aggression and self-injury (Buzan et al, 1998). In single-case studies with subjects with learning disability, risperidone - which blocks both $\mathrm{D}_{2}$ and serotonin type $2\left(5-\mathrm{HT}_{2}\right)$ receptors - seems to be effective in aggression (and has some effectiveness in attention-deficit hyperactivity disorder). In an add-on double-blind placebo-controlled crossover study of 37 institutionalised people with learning disability,Van den Borre et al (1993) found risperidone, in a daily dose of 4-12 mg, to be significantly better than placebo in controlling behavioural problems. The Aberrant Behavior Checklist and the Clinical Global Impression scale (Guy, 1976) were used to rate behavioural problems.

McDougle et al (1998) found risperidone to be effective in controlling aggression in a double-blind RCT of subjects with autism, confirming this drug's usefulness.

Serotonin receptor $\left(5-\mathrm{HT}_{2}\right)$ antagonism also occurs with these drugs. Olanzapine causes weight gain but also affects both $\mathrm{D}_{1-4}$ and $5-\mathrm{HT}_{2}$ sites, anecdotally, and in open- label trials (Potenza et al, 1999) is reported as effective in aggression and selfinjurious behaviour. Other atypical antipsychotics such as amisulpiride or quetiapine, which have respectively high $D_{3}$ affinity and low $D_{2}$ affinity, are anecdotally useful in autism, but it is unclear what direct relevance these site affinities have to prescribing. Neuroleptic adverse effects, and especially neuroleptic malignant syndrome, are a 
particular risk for people with learning disability (Boyd, 1993), especially in warmer climates and in hot summer.

The traditional neuroleptics are now rarely the drugs of first choice for the people with learning disability because of their sedative effects, EPS and epilepsy, and tardive dyskinesia. The atypical neuroleptics may also be more effective in treating behavioural and psychiatric disturbances.

Tools for assessing adverse effects generally target tardive dyskinesia or akathisia. There is no substitute, however, for careful review by the practitioner. The DISCUS (Dyskinesia Identification System Condensed User Scale) was developed specifically by Sprague et al (1989) for monitoring tardive dyskinesia in people with learning disability. Many types of professionals can be taught in a few hours to detect antipsychotic side-effects. Being female, being Asian, perinatal brain damage and early introduction of neuroleptics are predisposing factors for tardive dyskinesia. Patients on traditional (typical) antipsychotics with EPS have higher average $D_{2}$ occupancy compared with patients with no EPS. The optimal $D_{2}$ occupancy for the phenothiazines and butyrophenones is $70-80 \%$.

\section{Mood stabilisers}

Owing to the adverse effects of neuroleptic medications, non-neuroleptics such as lithium, carbamazepine, antidepressants, opiate antagonists, psychostimulants, beta-blockers, fenfluramine, etc. have also been tried for the treatment of behavioural problems in this population. In Spreat et al's (1989) study, $63 \%$ of people with learning disability showed a $>30 \%$ reduction in the frequency of aggressive behaviour when treated with lithium carbonate. There have also been case reports of improvement with lithium treatment in aggression and hyperactivity in adults without learning disability. Also in double-blind, placebo-controlled trials, lithium was effective in the treatment of aggression and hyperactivity.

Anticonvulsants, such as valproic acid and carbamazepine, are effective for rapidly cycling behavioural problems, including episodic dyscontrol. They are the treatment of choice where outbursts of rage are accompanied by abnormal electroencephalograms, but only carbamazepine is licensed in the UK for bipolar disorder. Sodium valproate is effective in epilepsy but contraindicated in active liver disease or a family history of liver disease. Liver function should be monitored at baseline and regularly thereafter, particularly during the first six months of treatment. Anticonvulsants also directly affect monoamines, for example, carbamazepine and valproate reduce dopamine turnover. There is no direct relationship between blood levels and response in affective disorders. Carbamazepine seems to have a prophylactic effect in bipolar disorder. Augmentation of lithium with carbamazepine is generally safe if introduced cautiously. Gabapentin is an anti-epileptic agent structurally related to $\gamma$-aminotbutyric acid (GABA). It is indicated as an add-on therapy in patients with resistant partial seizures. It does not require serum monitoring and may be useful for patients with biopolar disorder who are on multiple psychotropics. Some patients improve in mood even in the absence of seizure control. There are no RCTs of gabapentin in affective disorder. Porter et al (1999) advise that it be used as add-on therapy in cases where there is resistance to lithium, carbamazepine and valproate, or as a substitute when these agents are not tolerated.

Unlike carbamazepine and valproate, thioridazine is not teratogenic, nor is lamotrigine. There is anectodal evidence that lamotrigine can be helpful in mood disturbance.

Hyperactivity in people with more severe learning disability and in overactive disorder on the autistic spectrum (F84.4)(ICD-10; World Health Organization, 1992) is less susceptible to methylphenidate. If both methylphenidate and dexamphetamines fail, then clonidine, amantadine, pindolol and risperidone are all 'down the line' alternatives for activity disorders.

\section{Antidepressants}

Tricyclic antidepressants such as amitriptyline, nortriptyline, imipramine, clomipramine, etc. have been used to treat not only depressive disorders in the people with learning disability, but also behavioural problems such as aggression, hyperactivity and self-injurious behaviour. The association of aggression and self-injurious behaviour with psychiatric illness in people with severe and profound learning disability is very plausible. It has even been suggested by Davis et al (1997) that such behaviours should substitute for guilt and worthlessness in depression in people with severe learning disability. Clomipramine, in a double-blind, controlled crossover trial (Lewis et al, 1995), has been shown to be effective in stereotypies, and there is some evidence that perseverative behaviour may be reduced with selective serotonin reuptake inhibitors (SSRIs) (Branford et al, 1998). There has been evidence of fluoxetine's efficacy in treating selfinjurious behaviour in people with learning disability (Markowitz, 1992). Sovner et al (1993) also considered that fluoxetine acts as an antidepressant in these cases by treating an underlying depression. In some contradistinction to neuroleptics, 
antidepressants have only relatively recently been precisely prescribed to people with mental retardation, as people become more skilled in using the diagnostic tools mentioned above. The principle, again, is continuity prescribing as with people with normal intelligence. The use of SSRIs in learning disability practice is still based on open trials. There is no literature on the use with this population of serotonin and noradrenaline reuptake inhibitors (SNRIs), which do not have anticholinergic sideeffects; noradrenergic and specific serotonergic antidepressants (NASSA); or noradrenaline reuptake inhibitors (NARIs). Caution is necessary in the use of antidepressants with specific serotonergic action because of occasional emergent aggression, agitation and sleep disturbance.

The 5-HT 1 agonist buspirone in a daily dose of 20-50 mg may be effective in reducing challenging behaviour and increasing sociability.

\section{Opiate antagonists}

The use of opiate antagonists for the treatment of self-injurious behaviour is based on the hypothesis that there are excessive endogenous opioids in the system of persons who exhibit self-injurious behaviour, which increases their pain threshold, producing addiction to self-injury. Thus, antagonists would theoretically reduce self-injurious behaviour by blocking these opioids. The main drug used is naltrexone, which has a half-life of approximately 24 hours. Sandman (1988) in his review reported that approximately $65 \%$ of subjects treated showed some improvement in their selfinjurious behaviour, and suggested that beta endorphin plasma elevation, found immediately after an episode of self- injury, predicted a good response to naltrexone. Single-case studies support the idea that long-term naltrexone use may result in highly desirable reductions in self-injurious behaviour long after treatment ends, but Buzan et al (1995), reviewing 11 case reports of naloxone and 31 case reports of naltrexone, failed to establish the overall effectiveness of these agents. Buzan et al suggested that those who report that self-injurious behaviour makes them feel better, or who seek such behaviour without evidence of pain, are more likely to respond. There may be a therapeutic window effect.

\section{Acetylcholinesterase inhibitors}

There is no reason why these should be less effective than in the general population but there has been only one very small study to date (Kishnani et al, 1999).

\section{Anxiolytics}

High-dose beta-blockers are effective in controlling the somatic features of anxiety and might, therefore, have value in apprehensiveness in people with autism and prevent aggression. There is possibly a case for the use of beta-blockers in the treatment of the resistant aggressive patient. There is a collection of case reports, which suggest beta-blockers are useful in self-injurious behaviour.

\section{Drug reduction packages}

An RCT has been conducted using multiple measures, including direct observation, on a group of people with learning disability in whom psychotic illness had been excluded (Ahmed et al, 1999). The study showed that people with learning disability who do not have psychosis can be put on drug reduction programmes. A third experienced no ill effects after complete withdrawal, $20 \%$ experienced no ill effects with partial withdrawal, and the remainder had their medication reinstated without major problems. This multi-centre study provided some support for the syndrome of emergent dyskinesia as a complicating factor in drug withdrawal, and provided a useful basis for guidelines for drug reduction. Given the damage psychotropic medication does to individuals in the long term, as well as the cost of medication and the careful medical and nursing supervision it entails, such guidelines should be standard in the National Health Service. The researchers argue that attributes of carers is an important factor. Further studies are needed as to why people might reintroduce antipsychotic medication. The best predictors of which people with learning disability would fail on the drug reduction programme were environmental - such as lack of training of professional care-givers, lack of refresher courses, skill levels of the care-givers in general and the quality of the environment in which the behaviourally disturbed person with learning disability was living. Finally, having considered care-giver factors, in patients without firm psychiatric diagnosis, one ought to attempt cautiously to reduce neuroleptic medication by $25 \%$ per month.

\section{Conclusions}

Therapies for people with learning disabilities, as for children, lack sufficient proper rigorously controlled trials. At present, psychopharmacology for people with learning disability is largely based on an extrapolation from adult populations of 
Box 3. Key messages

Evidence-based medicine is in its infancy in the field of learning disability

The clinicians cannot expect support in prescribing from the literature

On introducing psychotropic medication for behavioural problems, the clinician should have considered exit strategies from such drugs

People with learning disabilities who are on psychotropic medication for behavioural reasons ought to be considered for drug reduction programmes

The factors which predict failure of drug reduction programmes are principally behavioural

average intellect. People with learning disabilities need studious attention to their special needs. Behavioural problems of unknown causes often result in trial-and-error prescribing.

\section{References}

Ahmed, Z., Fraser, W., Kerr M., et al (1999) The effects of reducing antipsychotic medication in people with a learning disability. British Journal Psychiatry, in press.

Aman, M. G., Singh, N. N., Stewart, A.W., et al (1985) The aberrant behavior checklist: a behavior rating scale for the assessment of treatment effects. American Journal of Mental Deficiency, 89, 485-491.

Bishop, A. (1992) Empirical approach to psychopharmacology for institutionalized individuals with severe or profound retardation. Mental Retardation, 30, 283-288.

Boyd, R. D. (1993) Neuroleptic malignant syndrome and mental retardation. Review and analysis of 29 cases. American Journal on Mental Retardation, 98, 143-155.

Branford, D., Bhaumik, S. \& Naik, B. (1998) Selective serotonin re-uptake inhibitors for the treatment of perseverative and maladaptive behaviours of people with intellectual disability. Journal of Intellectual Disability Research, 42, 301-306.

Brylewski, J. \& Duggan, L. (1998) Antipsychotic Medication for Challenging Behaviour in People with Learning Disability. The Cochrane Library. Oxford: Update Software.

Buzan, R. D., Thomas, M., Duborsky, S. L., et al (1995) The use of opiate antagonists for treatment of self injurious behaviour. Journal of Neuropsychiatry and Clinical Neuroscience, 7, 437-444.

- Dubovsky, S. L., Firestone, D., et al (1998) Use of clozapine in 10 mentally retarded adults. Journal of Neuropsy and Clinical Neurosciences, 10, 93-95.

Clarke, D. J., Kelley, S., Thinn, K., et al (1990) Psychotropic drugs and mental retardation: 1 . Disabilities and the prescription of drugs for behaviour and for epilepsy in three residential settings. Journal of Mental Deficiency Research, 34, 385-395.

Davis, J. P., Judd, F. K. \& Herrman, H. (1997) Depression in adults with intellectual disability. Part 1: A review. Part 2: A pilot study. Australian and New Zealand Journal of Psychiatry, 31, 232-242; 243-251.
Deb, S. \& Fraser W. (1994) The use of psychotropic medication in people with learning disability. Towards rational prescribing. Human Psychopharmacology, 9, 219-272.

Duggan, L. \& Brylewski, J. (1999) Effectiveness of antipsychotic medication in people with intellectual disability and schizophrenia. A systematic review. Journal of Intellectual Disability Research, 43, 94-105.

Einfeld S. L. (1990) Guidelines for the use of psychotropic reduction in individuals with developmental disorders and development disabilities. Australia and New Zealand Journal of Developmental Disability, 161, 71-73.

Guy, W. (1976) ECDEU Assessment Manual for Psychopharmacology. Revised DHEW Pub. (ADM). Rockville, MD: National Institute for Mental Health.

Healy, D. \& Nutt, D. (1998) Prescriptions, licences and evidence. Psychiatric Bulletin, 22, 680-684.

Kishnani, P. S., Sullivan, J. A., Walter, B. K, et al (1999) Cholinergic therapy for Down's syndrome. Lancet, 353 1064-1065.

Lewis, M., Bodfish, J., Powell, S., et al (1995) Clomipramine treatment for stereotyped and repetitive movement disorders association with mental retardation. American Journal on Mental Retardation, 100, 299-312.

Markowitz, P. (1992) Effect of fluoxetine on self-injurious behavior in the developmentally disabled: a preliminary study. Journal of Clinical Psychopharmacology, 12, 27-31.

Matson, J. (1988) The Psychopathology Instrument for Mentally Retarded Adults. Illnois: International Diagnostic Systems.

McDougle, C. J., Holmes, J. P., Carlson, D., et al (1988) A double-blind, placebo-controlled study of risperidone in adults with autistic disorder and other pervasive developmental disorders. Archives of General Psychiatry, 55, 633-641.

Moss, S. C., Ibbotson, B. \& Prosser, H. (1994) The Psychiatric Assessment Schedule for Adults with Developmental Disability (The PAS-ADD); Interview Development and Compilation of the Clinical Glossary. Manchester: Hester Adrian Research Centre, University of Manchester.

-, Patel, P., Prosser, H., et al (1997) Psychiatric morbidity in older people with moderate and severe learning disability 1: Development and reliability of the patient interview (PAS-ADD). British Journal of Psychiatry, 163, 471-480.

Porter, R., Ferrier, N. \& Ashton, H. (1999) Anticonvulsants as mood stabilisers. Advances in Psychiatric Treatment, 5, 96-103.

Potenza, M. N., Holmes, J. P., Kanes, S .J., et al (1999) Olanzapine treatment of children, adolescents, and adults with pervasive developmental disorders; an open label pilot study. Journal of Clinical Psychopharmacology, 19, 37-44.

Reiss, S. (1988) The Reiss Screen Test Manual. Orlando, IL: International Diagnostic Systems.

Sandman, C. A. (1988) B-endorphin disregulation in autistic and self-injurious behaviour: a neurodevelopmental hypothesis. Synapse, 2, 193-199.

Sovner, R., Fox, C. J., Lowry, M. J., et al (1993) Fluoxetine treatment of depression and associated self-injury in two adults with mental retardation. Journal of Intellectual Disability Research, 37, 301-311.

Sprague, R. L., Kalachnik, J. E., Slaw, K. M., et al (1989) Psychometric properties of the Dyskinesia Identification System: Condensed User Scale (DISCUS). Mental Retardation, 27, 141-148.

Spreat, S., Behar, D., Reneski, B., et al (1989) Lithium carbonate for aggression in mentally retarded persons. Comprehensive Psychiatry, 30, 505-511.

Thompson, C. (1994) The use of high dose antipsychotic medication. Consensus statement. British Journal of Psychiatry, 164, 448-458.

Van den Borre, R., Vermote, R., Butiens, M., et al (1993) Risperidone as add-on therapy in behavioural disturbances in mental retardation: a double-blind placebo-controlled cross-over study. Acta Psychiatrica Scandinavica, 87, 167171.

World Health Organization (1992) The ICD-10 Classification of Mental and Behavioural Disorders. Geneva: WHO. 


\section{Multiple choice questions}

1. There is an adequate evidence base for the use of psychopharmacology:

a in aggressive behaviour disturbance in people with learning disability

$\mathrm{b}$ in schizophrenia in people with learning disability

$c$ in self-injurious behaviour in people with learning disability

$\mathrm{d}$ in major depression in borderline learning disability

e in episodic dyscontrol in people with learning disability.

2. Psychotropic drug reduction packages:

a may commonly precipitate major relapses in behaviour in people with challenging behaviour

b may result in emergent dyskinesia

c can proceed at $25 \%$ medication reduction per month

d are unaffected by the skill level of the immediate care-givers

e will demonstrate clinical awareness of the wider various features of untargeted prescribing.

3. In non-specific behavioural disorders:

a beta-blockers can be the principal drug of choice

b antidepressants may play a useful role

c lithium may be considered

d anticonvulsants may be the drug of choice

e carbamazepine may have a prophylactic effect.
4. When schizophrenia is diagnosed in people with learning disability:

a the traditional neuroleptics remain the drugs of first choice

b the DISCUS is a first choice way of assessing tardive dyskinesia in people with learning disability

c the DISCUS does not require any training

d diagnostic support from a recognised standardised instrument is desirable

e the scientific literature will provide clear trial evidence for use of medication.

5. Overactivity in people with severe learning disability:

$a$ is best managed by methylphenidate or dexamphetamine

b is best managed by naltrexone if there is complicating self-injurious behaviour

c requires a baseline assessment involving environmental factors

$\mathrm{d}$ may be alleviated by clozapine if there is complicating self-injurious behaviour

e risperidone may be helpful if there is accompanying aggression.

\section{MCQ answers}

\begin{tabular}{|c|c|c|c|}
\hline 1 & 2 & 3 & 4 \\
\hline$F$ & a F & a $F$ & a 1 \\
\hline$F$ & b $\mathrm{T}$ & b $\mathrm{T}$ & b \\
\hline$F$ & c $T$ & c $\mathrm{T}$ & c 1 \\
\hline$T$ & d F & d $T$ & d \\
\hline F & e $T$ & e $T$ & e \\
\hline
\end{tabular}

